Two simple methods that are clinically useful for analyzing impaired memory and learning are selective reminding or restricted reminding. These new methods provide simultaneous analysis of storage, retention, and retrieval during verbal learning because they let the patient show learning by spontaneous retrieval without confounding by continual presentation. Because selective reminding and restricted reminding let the patient show consistent retrieval without any further presentation, they also distinguish list learning from item learning, so that impaired memory and learning can be analyzed further in terms of two stages of learning (item and list).

\title{
Evaluating storage, retention, and retrieval in disordered memory and learning
}

HERMAN BUSCHKE, M.D., and PAULA ALTMAN FULD, Ph.D.

T he purpose of this paper is to present two simple methods for clinical use in evaluating memory and learning. These new methods ${ }^{1}$ allow us to analyze simultaneously initial storage, retention, and retrieval from long-term storage as the patient learns to recall a list of words, so that we can understand the nature of the patient's impaired memory and learning.

To understand what happens during such learning in terms of storage, retention, and retrieval, it is necessary to let the patient show what he has learned by having him spontaneously recall items without further presentation. To show initial storage, and to show retention in longterm storage despite recall failure, ${ }^{2}$ it is necessary to show that the patient can recall spontaneously such items without presentation. Therefore, evaluation of initial storage and subsequent retention in long-term storage both depend on spontaneous retrieval from long-term storage. Spontaneous retrieval without further presentation also is necessary to distinguish true retrieval from long-term

From the Saul R. Korey Department of Neurology and Rose F. Kennedy Center for Research in Mental Retardation and Human Development and the Department of Neuroscience, Albert Einstein College of Medicine, Bronx, New York.

This study was supported by USPHS Grants MH-17733 to H.B. from NIMH, NS-03356 from NIMS, and HT-01799-07 from NICHD.

Received for publication July 8, 1974.

Requests for reprints and test forms should be sent to $H$. Buschke, M.D., The Saul R. Korey Department of Neurology, Albert Einstein College of Medicine, 1300 Morris Park Avenue, Bronx, NY 10461. storage from the patient's immediate recall of items that have just been presented (recall from short-term storage). ${ }^{3-9}$ Thus, it is crucial to provide the patient with adequate opportunity to demonstrate learning by multiple recall trials without further presentation, to understand impaired memory and learning in terms of initial storage, retention, and retrieval. The methods of selective reminding and restricted reminding presented here do allow the patient to show spontaneous retrieval without further presentation, so that we can analyze a patient's disordered memory and learning simultaneously in terms of storage, retention, and retrieval during verbal learning.

In both of these methods the patient is simply asked to learn a list of words by verbal recall in any order. The entire list is read aloud once to the patient at a 2 -second rate before his first recall attempt. The patient then tries to recall all of the words in the list in any order (free recall). In selective reminding the patient is selectively reminded only of those items he did not recall on the immediately preceding trial, before he again attempts to recall all items in the list. This procedure of selective reminding is continued throughout learning and allows the patient to show that he has learned some of the items just recalled, by spontaneously retrieving them again without presentation on the next trial. Such selective reminding also may maximize learning by directing the patient's attention to just those items he did not recall on the previous attempt. 


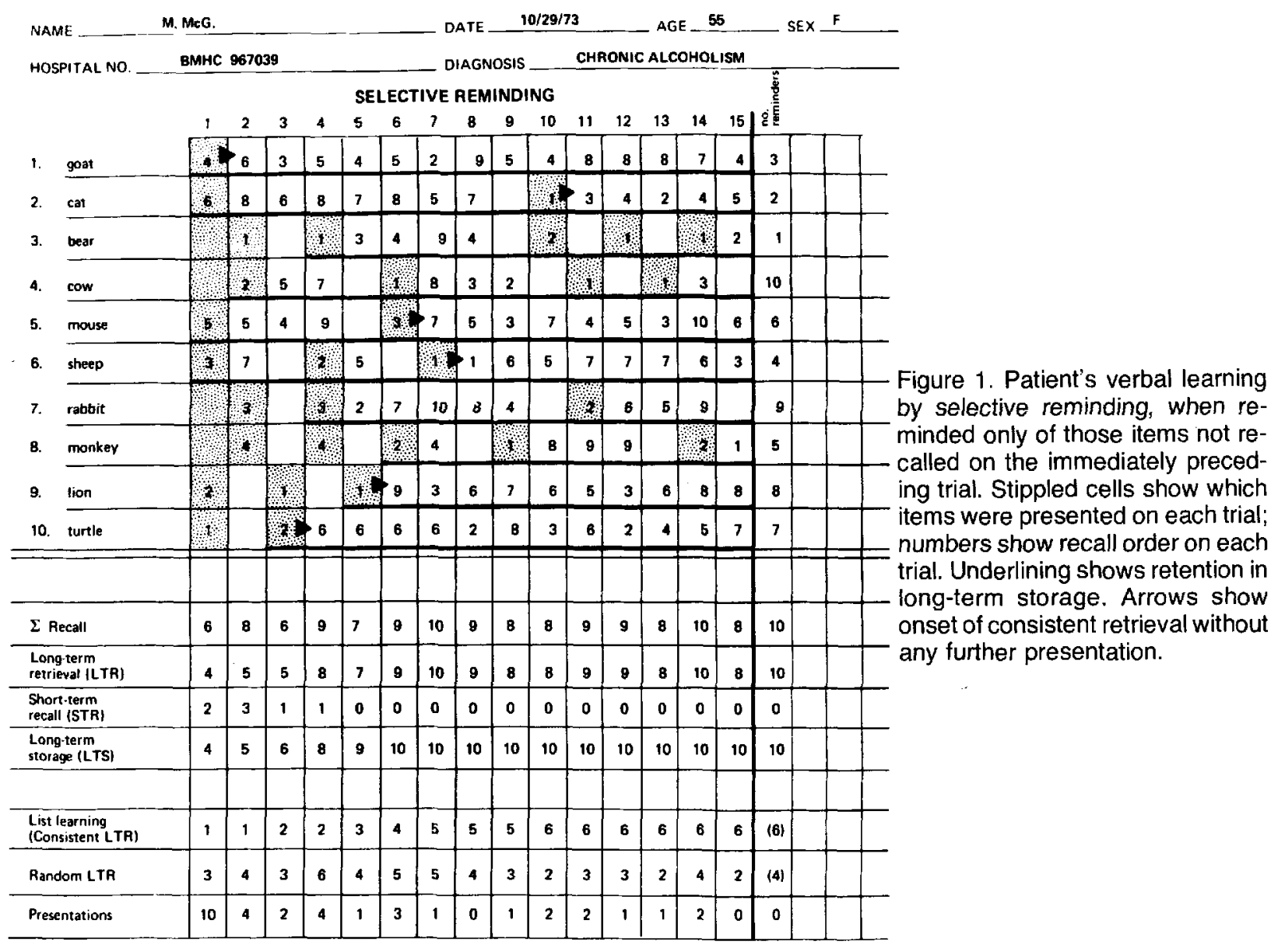

In restricted reminding the patient is also asked to learn a list of words by verbal recall in any order. The entire list is read aloud once to the patient at a 2 -second rate before his first attempt to recall all of the words in the list in any order. Then the patient is reminded of any words not yet recalled before his next attempt to recall all of the items in the list. Since this procedure of restricted reminding is continued only until each item in the list has been recalled just once, in order to show that the patient has at least attended to all items in the list, the patient can show learning by spontaneous recall without any further presentation at all after the initial recall of each item. Such restricted reminding allows concurrent evaluation of initial storage, retention, and spontaneous retrieval from long-term storage during learning, without any confounding by continuing presentation. Restricted reminding provides the strongest estimate of initial storage, since any subsequent spontaneous retrieval of an item without any further presentation at all shows initial storage on (or before) the trial when that item was last presented. Similarly, spontaneous retrieval of an item without any further presentation after recall failure shows retention of that item in long-term storage, and demonstrates that those recall failures reflect retrieval failure, rather than loss from storage (retention failure).

Thus, in both methods the patient is asked to make repeated attempts to recall all items (in any order) from a short list that is read aloud to him. To evaluate retrieval from long-term storage during such free recall verbal learning, the patient is allowed to recall items without further presentation before subsequent recall attempts. In selective reminding, no items are presented that the patient has just recalled; i.e., he is selectively reminded only of those items he did not recall on the immediately preceding trial. In restricted reminding, an item is never presented again after it has once been recalled; i.e., each item is presented only until it has been recalled just once. Since these methods for evaluating memory and learning clearly depend on retrieval by the patient, it is important to give the patient enough time and encouragement on each recall attempt to obtain the maximal retrieval necessary for accurate evaluation of initial storage, subsequent retention, and retrieval from long-term storage.

The clinical use of these complementary methods is illustrated by their application in analyzing the impaired memory and learning of a patient with chronic alcoholism. This patient is a 55-year-old woman who worked as an accountant until shortly before she was hospitalized because of flaccid quadriparesis. She gave a 10 -year to 15-year history of drinking at night after going without food all day. She has biopsy-proved alcoholic cirrhosis and hepatitis and has had previous upper-gastrointestinal bleeding. Although her quadriparesis may reflect alcoholic polyneuropathy, an elevated cerebrospinal fluid 


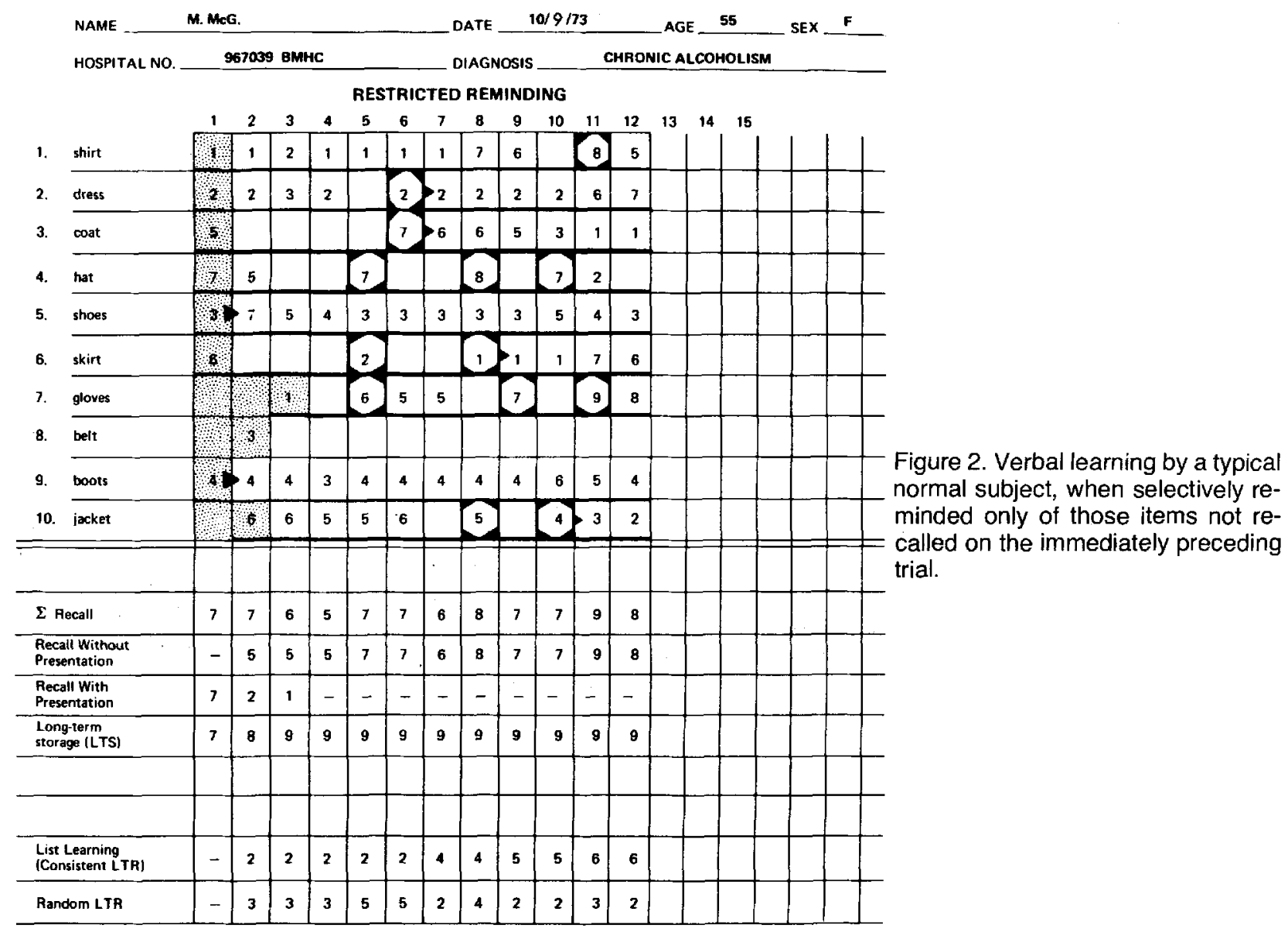

protein as high as $400 \mathrm{mg}$ with few cells suggests Guillain-Barré. Her electroencephalogram was mildly and diffusely slow, but she was alert, oriented, and cooperative.

Selective reminding. Figure 1 shows this patient's learning by selective reminding. The patient was asked to learn this list of 10 animals by verbal recall in any order. The entire list was read aloud to the patient at a 2 -second rate before her first recall attempt. Thereafter the patient was selectively reminded only of those items that she had not recalled on the immediately preceding trial. Since the patient tried to recall all of the items on each recall attempt, this allowed her to show what she had learned by spontaneously recalling items without further presentation. The stippled cells show which items were presented on each trial (i.e., those items that were not recalled on the immediately preceding trial). The numbers indicate the order in which she recalled those items that were recalled on each trial; if there is no number in a cell, it means that item was not recalled on that trial.

Recall without further presentation indicates retrieval from long-term storage. ${ }^{3-9}$ For example, "goat" was retrieved from long-term storage on trial 2 , since it was recalled without presentation on that trial. This item was retrieved from long-term storage because it was recalled even though the presentation and recall of other items interfered with the short-term retention of this item. The heavy underlining indicates retention in long-term storage. Since "goat" was retrieved from long-term storage on trial 2 , information about this item must have entered long-term storage on the previous trial.

Continuing retention in long-term storage (even after recall failures) is shown by continuing the underlining after an item has once been retrieved from long-term storage. This assumes that recall failures represent retrieval failure, rather than retention failure or loss from storage. This assumption appears to be valid for normal adults and children;2,10 it will be shown to be valid for this specific patient also in that she retrieved items again spontaneously after recall failure, without any further presentation.

To simplify the scoring of retrieval from long-term storage, and to obtain a reasonable estimate of retrieval from long-term storage on the first recall trial, all underlined recall is counted as retrieval from long-term storage, even when an item is presented again because of previous recall failure. Since it can be shown that items remain in storage despite failure to recall them, ${ }^{2}$ it seems reasonable to regard such presentations after initial storage simply as reminders. Items not retrieved from long-term storage are recalled from short-term storage; ${ }^{3-9}$ these are items that 
were recalled only with presentation and have not yet been underlined, such as "turtle" on trial 1.

The arrows show the trial after which an item was consistently retrieved from long-term storage on all subsequent recall attempts without any further presentation at all. This separates retrieval from long-term storage into consistent retrieval and inconsistent or random retrieval. The significance of such consistent retrieval from longterm storage will be discussed later.

By allowing the patient to show retrieval from longterm storage by recall without further presentation, selective reminding separates retrieval from long-term storage and recall from short-term storage, estimates long-term storage, and separates retrieval from long-term storage into consistent and random retrieval. Comparison of this patient's learning (figure 1) with that of a normal adult (figure 2) indicates that this patient's verbal memory and learning are quite impaired. This patient continued to need reminders for many trials and never was able consistently to recall all of the list without further presentation. She required six trials before all 10 items were stored, and then could not retrieve all of the items retained in longterm storage. Even after 10 trials she could retrieve only six items consistently on all later trials without any further reminders. This analysis of her learning by selective reminding seems to indicate that this patient had continuing retrieval difficulty as well as some impairment of initial storage.
Restricted reminding. Figure 3 shows this patient's learning by restricted reminding. The patient was asked to learn a different list of 10 items of clothing by verbal recall in any order. The entire list was read aloud to the patient at a 2-second rate before her first recall attempt. Thereafter the patient was reminded only of those items that she had not yet recalled at all, until each item was recalled just once. After each item was recalled once, it was never presented again. Since all items were recalled at least once by the third trial, there were no further presentations at all after this patient's third recall attempt. Since the patient tried to recall all items in the entire list on each recall attempt, this allowed the patient to show learning by recalling items without presentation, to show initial storage, retention, and retrieval from long-term storage without confounding by any further presentation. The stippled cells show which items were presented on each trial (i.e., those items that had not been yet recalled at all). The numbers indicate the order in which she recalled those items that were recalled on each trial; if there is no number in a cell, it means that item was not recalled on that trial.

Retrieval from long-term storage is shown by spontaneous recall without any further presentation after the initial recall of an item. For example, "shirt" was retrieved from long-term storage for the first time on trial 2 , while "coat" was not retrieved from long-term storage for the first time until trial 6. However, information about both "shirt" and "coat" must have entered long-term

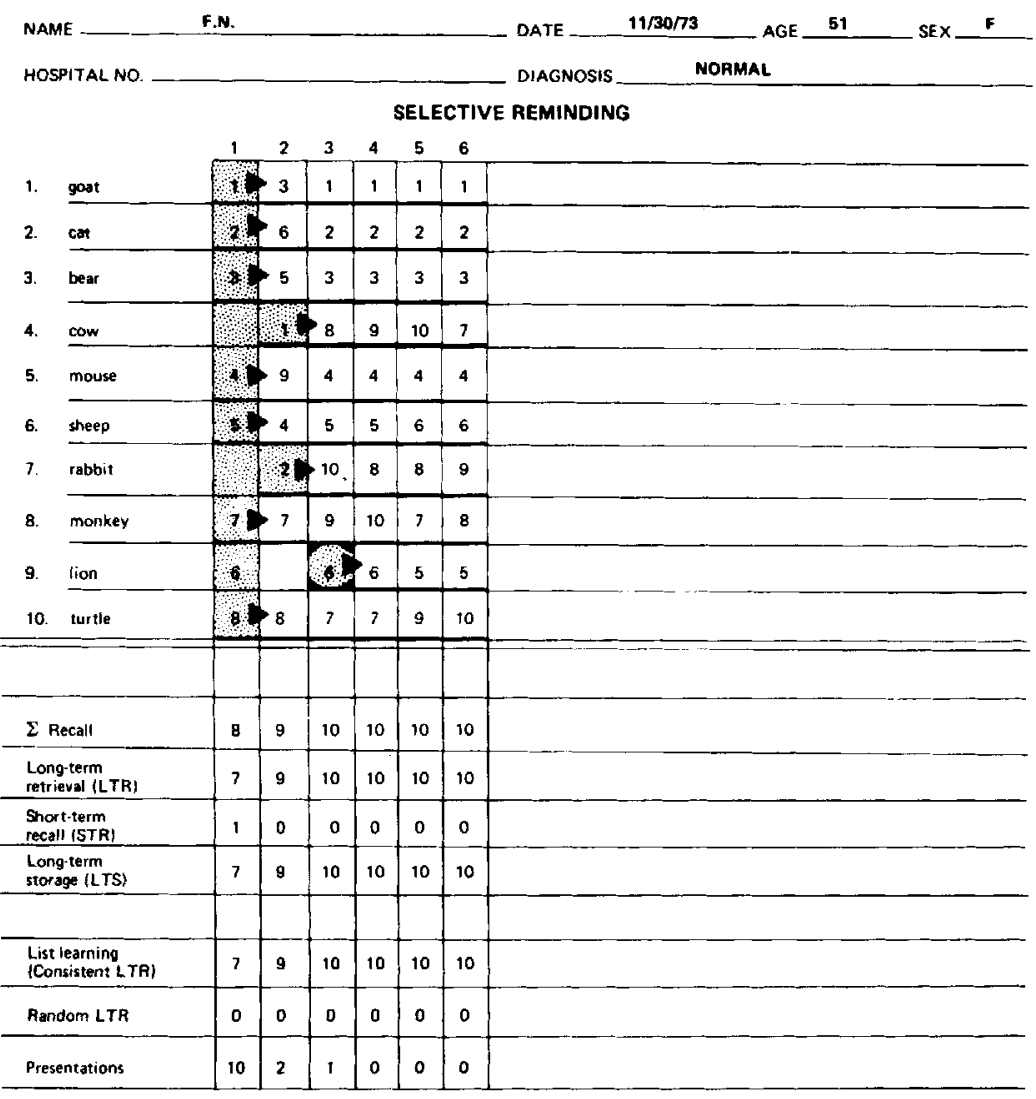

Figure 3. Patient's verbal learning by restricted reminding, when reminded of each item only until it has been recalled once. 
storage on trial 1, since they were not presented again after trial 1 (when both were recalled for the first time).

Retention in long-term storage is indicated by the heavy underlining. Since all items except for "belt" eventually were retrieved spontaneously by this patient, without any further presentation at all after initial recall of each item, it appears that the minimal presentation provided by restricted reminding was sufficient for initial storage of at least nine items.

Continuing retention in long-term storage is shown by continuing the heavy underlining after an item has once been retrieved from long-term storage. The boxed numbers indicate spontaneous retrievals without any further presentation after recall failures, showing that those recall failures were caused by retrieval failure (rather than by retention failure or loss from storage). This confirms the assumptions used in analyzing this patient's learning by selective reminding: Information about items remain in long-term storage and recall failures represent retrieval failure.

The arrows show the trial after which an item was consistently retrieved from long-term storage on all subsequent recall attempts without any further presentation. This separates retrieval from long-term storage into consistent retrieval and inconsistent or random retrieval. Figure 4 (left), which summarizes this patient's leaming by restricted reminding, shows that much of this patient's retrieval from long-term storage was not consistent. Although information about 9 of the 10 items was encoded (and retained) in long-term storage, her retrieval of the items retained in long-term storage was limited and inconsistent. Her inconsistent retrieval from long-term storage is reflected in the cumulative number of recall failures shown in figure 4 (right), which also shows that almost all of these recall failures were recovered spontaneously. This indicates that her recall failures were retrieval failures. It appears that while this patient had some impair- ment of initial storage, her retention in long-term storage was good. Most of her difficulty in learning to recall this 10-item list apparently was caused by the impaired retrieval.

Discussion. Both selective reminding and restricted reminding showed that this patient's impairment of learning was caused mostly by her impaired retrieval from longterm storage. Restricted reminding confirmed the findings of selective reminding: Although initial storage appeared to be somewhat impaired, retention in long-term storage was intact, and her recall failures were caused by retrieval failure. Although relatively short lists of items from a single category were used with this patient to facilitate retrieval, longer lists of unrelated items also can be used to evaluate impaired memory and learning.

In addition to evaluating the storage and retrieval of individual items, both of these methods aiso can provide an evaluation of the patient's ability to learn the list as a list. ${ }^{1}$ We would say ordinarily that a patient has learned the entire list when he can consistently recall all of the items in that list on every recall attempt, without any further presentations or reminders at all. Therefore it should follow, for example, that when a patient can consistently recall the same five items from a 10 -item list on all recall attempts (without any further presentation of those items), the patient has learned a five-item list or 50 percent of the 10-item list. Thus, consistent recall of an item on all subsequent recall attempts without any further presentation should indicate that the item has been learned as part of the list (so that retrieval of that item has been integrated with retrieval of other consistently recalled items), and the number of items consistently retrieved after each recall trial should indicate how much of the list has been learned at that point. Although this patient learned 10 items by selective reminding and nine items by restricted reminding, her consistent retrieval indicates

Name: M.MCG. Female Age 55 Date: 9 October 1973

Test: Verbal - 10 Clothes Restricted Reminding ID: $967039 \mathrm{BMHC}$
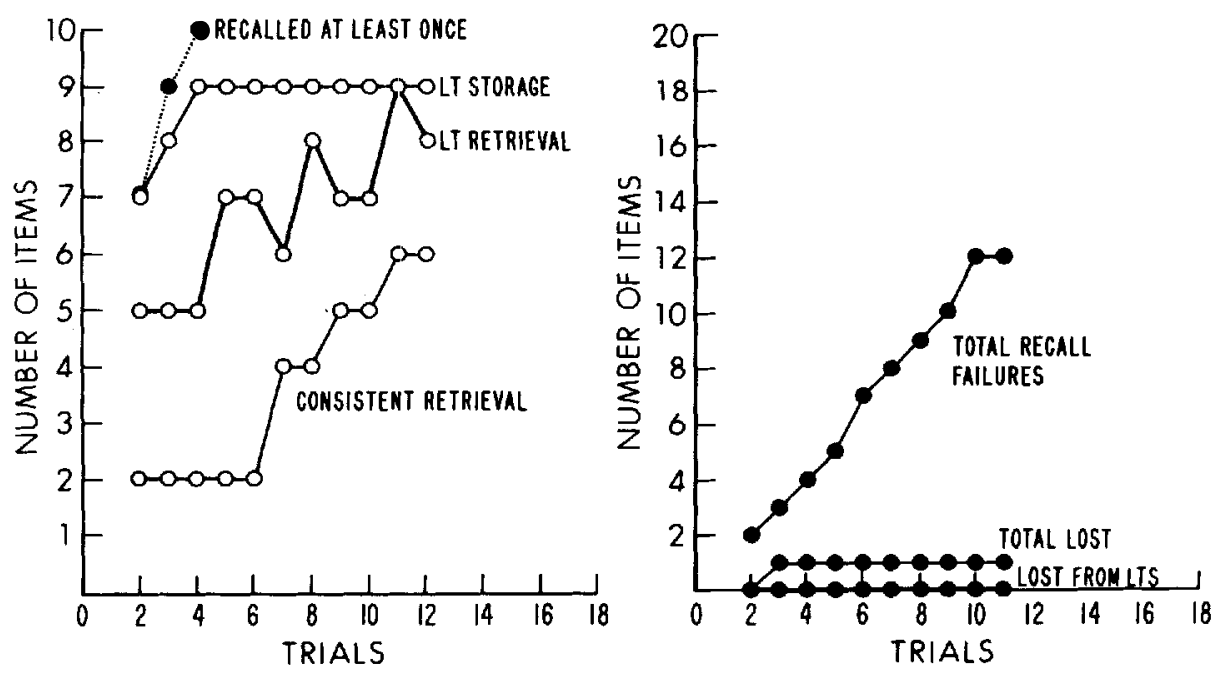

Figure 4. Patient's storage, retention, and retrieval during verbal learning by restricted reminding (left). Cumulative total of all recall failures and of those not spontaneously retrieved again (right). 
Nome: M.McG. Female Age 55. Dare: 9 October 1973

Test: Verbal-10 Clothes Restricted Reminding ID: 967039 BMHC
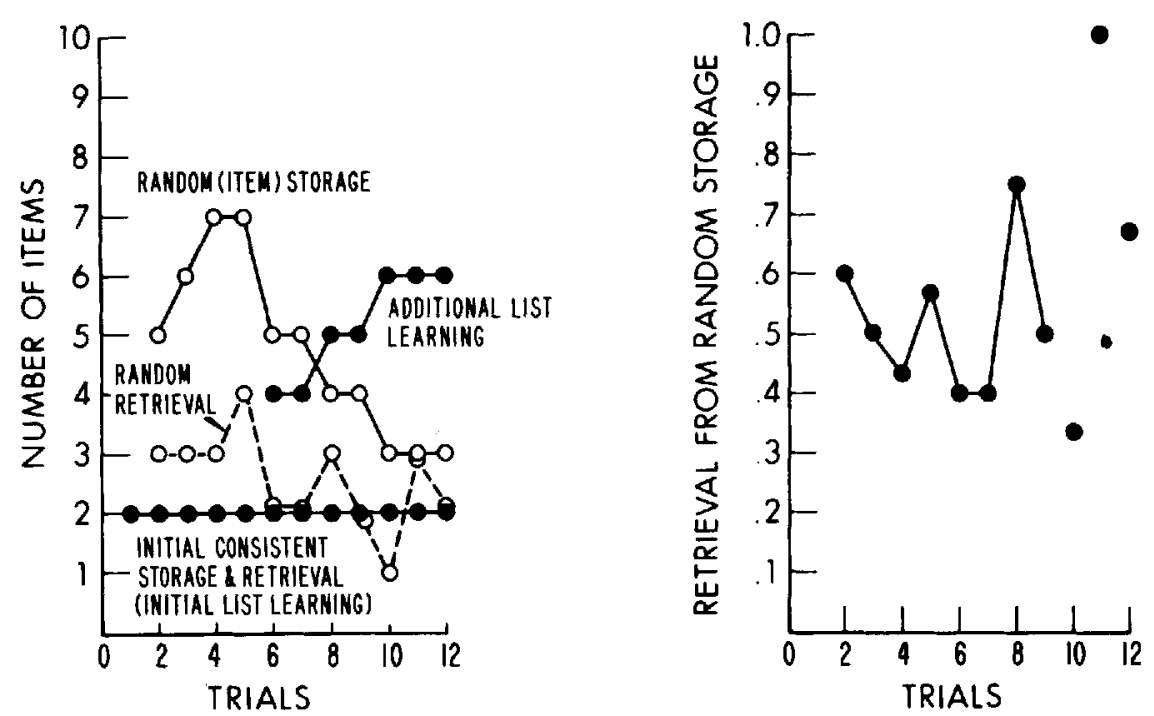

Figure 5. Analysis of patient's learning by restricted reminding in terms of stages of random retrieval (item learning) and consistent retrieval (list learning) from long-term storage, to show random (item) storage, nonincremental retrieval from random storage, initial consistent storage and retrieval (initial list learning), and transition from random to consistent retrieval (additional list learning). Right panel shows nonincremental probability of random retrieval from long-term storage prior to onset of consistent retrieval.

that she had learned only six items as part of the list (or 60 percent of the list) after 12 trials.

The contention that random and consistent retrieval from long-term storage reflect qualitatively different stages of item and list learning, respectively, is supported by recent work ${ }^{11 a}$ that shows that retrieval from longterm storage does not improve progressively until consistent retrieval is achieved, but rather shows that the probability of retrieval from long-term storage remains relatively constant prior to the abrupt onset of consistent retrieval. This means that recall improves during such free recall verbal learning because information about more and more items reach the second stage of list learning characterized by perfect retrieval, because the retrieval of such items has been integrated with the retrieval of other consistently retrieved items and not because the "strength" of an item increases in long-term storage until perfect retrieval is achieved. ${ }^{11 b}$ The relatively constant probability of random retrieval from longterm storage by this patient before the onset of consistent retrieval is shown in figure 5 (right). This nonincremental random retrieval from long-term storage before the abrupt onset of consistent retrieval justifies analysis of this patient's impairment of memory and learning in terms of two stages ${ }^{12-15}$ of learning (item and list). The components of such a two-stage analysis of learning include: The number of items in random (item) storage for inconsistent retrieval, the number of items randomly retrieved from such (item) storage, the number of items initially retained in the second stage (list) of learning for consistent retrieval from the onset of learning (initial list learning), and the number of items transferred from the first stage (item) of learning to the second stage (list) of learning for consistent retrieval (additional list learning). Figure 5 (left) shows such an analysis of this patient's learning by restricted reminding. This patient's initial list learning was very low, since she was able to retrieve only two items consistently from the beginning. Until the sixth trial, most items were retained in the first stage of random (item) storage, from which she randomly retrieved a relatively constant proportion on each trial. Not until the sixth trial was there additional list learning, when the number of items retained in the first stage (item) of learning began to decrease as information about items was transferred from the first to the second stage (list) of learning for consistent retrieval on all subsequent recall attempts. Even after 12 trials only six of the nine items retained in long-term storage had reached the second stage of learning, while three items still remained in the first stage (item) of learning.

These new methods are useful in analyzing disorders of memory and learning because they allow the patient to show learning by spontaneous retrieval without further presentation. Restricted reminding will show retention and retrieval without confounding by further presentation and can provide the most accurate estimate of initial storage (by eventual recall without any further presentation). Selective reminding will provide enough presentation for maximal learning and may direct attention to items not yet learned. While both methods require the use of "extended recall," by encouraging the patient to recall as much as possible on each recall attempt in order to obtain the maximum recall necessary for accurate evaluation of storage, retention, and retrieval, this is especially important in restricted reminding. ${ }^{2}$

\section{Acknowledgments}

We thank David Drachman, Simon Horenstein, Robert Katzman, Marcel Kinsboume, and Steven Mattis for helpfuł discussion. 


\section{REFERENCES}

1. Buschke $H$ : Selective reminding for analysis of memory and learning. $J$ Verbal Learning and Verbal Behavior 12:543-550, 1973

2. Buschke $\mathrm{H}$ : Spontaneous remembering after recall failure. Science 184:579-581, 1974

3. Craik FIM: Two components in tree recall. J Verbal Learning and Verbal Behavior 7:996-1004, 1968

4. Glanzer $M$, Cunitz AR: Two storage mechanisms in free recall. $J$ Verbal Learning and Verbal Behavior 5:351-360, 1966

5. Glanzer $\mathrm{M}$ : Storage mechanisms in recall. In Bower GH (Editor): Psychology of Learning and Motivation. New York, Academic Press, 1972, vol 5, pp 129-193

6. Tulving $\mathrm{E}$ : Intratrial and intertrial retention: Notes toward a theory of free recall verbal learning. Psychol Fev 71:219-237, 1964

7. Tulving $E$, Colotla VA: Free recall of tri-lingual lists. Cognitive Psychol $1: 86-98,1970$

8. Waugh NC, Norman DA: Primary memory. Psychol Rev 72:89-104, 1965
9. Baddeley AD, Warrington EK: Amnesia and the distinction between long and short-term memory. $J$ Verbal Learning and Verbal Behavior 9:176-189, 1970

10. Buschke $\mathrm{H}$ : Components of verbal learning in children: analysis by selective reminding. J Exp Child Psychol (In press)

11a. Buschke $H$ : Two stages of learning by children and adults. Bull Psychonom Soc (In press)

11b. Restle $F$ : Significance of alt-or-none learning. Psychol Bull 64:313-325, 1965

12. Waugh NC, Smith JEK: A stochastic model for free recall. Psychometrika 27:141-152, 1962

13. Bower $\mathrm{GH}$, Theios $\mathrm{J}$ : A learning model for discrete performance levels. in Atkinson RC (Editor): Studies in Mathematical Psychology. Stanford, CA, Stanford University Press, 1963, pp 1-32

14. Kintsch W, Morris CJ: Application of a Markov model to free recall and recognition. J Exp Psychol 69:200-206, 1965

15. Evans $R B$, Dallenback KM: Single-trial learning: A stochastic model for the recall of individual words. Am J Psychol 78:545-556, 1965 


\section{Neurology}

\section{Evaluating storage, retention, and retrieval in disordered memory and learning}

HERMAN BUSCHKE and PAULA ALTMAN FULD

Neurology 1974;24;1019

DOI 10.1212/WNL.24.11.1019

This information is current as of November 1, 1974

\section{Updated Information \& \\ Services}

Citations

Permissions \& Licensing

Reprints including high resolution figures, can be found at: http://n.neurology.org/content/24/11/1019.full

This article has been cited by 28 HighWire-hosted articles:

http://n.neurology.org/content/24/11/1019.full\#\#otherartic les

Information about reproducing this article in parts

(figures,tables) or in its entirety can be found online at: http://www.neurology.org/about/about_the_journal\#permi ssions

Information about ordering reprints can be found online: http://n.neurology.org/subscribers/advertise

Neurology ${ }^{\circledR}$ is the official journal of the American Academy of Neurology. Published continuously since 1951, it is now a weekly with 48 issues per year. Copyright (O) 1974 by the American Academy of Neurology. All rights reserved. Print ISSN: 0028-3878. Online ISSN: 1526-632X.

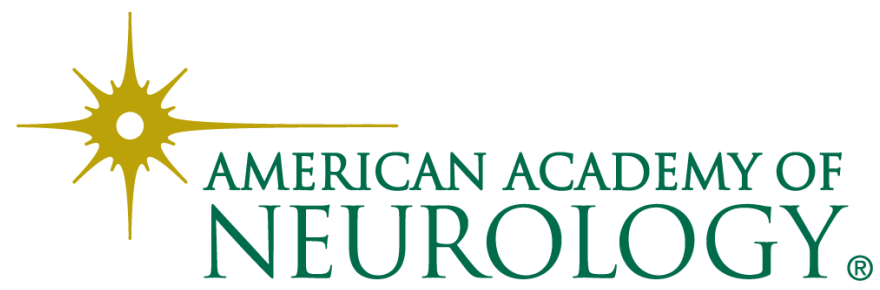

